Check for updates

Cite this: RSC Adv., 2018, 8, 15725

Received 21st November 2017 Accepted 14th March 2018

DOI: 10.1039/c7ra12659

rsc.li/rsc-advances

\section{Identification of absorbed constituents and evaluation of the pharmacokinetics of main compounds after oral administration of yindanxinnaotong by UPLC-Q-TOF-MS and UPLC-QqQ-MS†}

\author{
Leilei Gong, (D) a Haiyu Xu, ${ }^{a}$ Huijun Yuan, ${ }^{\mathrm{b}}$ Lan Wang, ${ }^{a}$ Xiaojie Yin, ${ }^{a}$ Moqi Fan, ${ }^{\mathrm{c}}$ \\ Long Cheng, (D) ${ }^{d}$ Xiaojing Ma, ${ }^{a}$ Rixin Liang ${ }^{\star a}$ and Hongjun Yang ${ }^{\star a}$
}

Yindanxinnaotong capsule (YDXNT), a traditional Chinese formula, has been used to treat cardiocerebrovascular diseases for several decades. Previous research has focused on evaluating the pharmacological properties and main compounds of YDXNT in vitro and in vivo. However, the multiple bioactive compounds in vivo remain poorly understood. In the present research, an integrative strategy using UPLC-Q-TOF-MS combined with UPLC-QqQ-MS was employed to detect the absorbed constituents and investigate the pharmacokinetics of main compounds in the plasma after oral administration of YDXNT. UPLC-Q-TOF-MS was developed to detect the absorbed constituents and their metabolites in the plasma after oral administration in rats. A total of 52 constituents, including 44 prototype compounds and 8 metabolites, were identified or tentatively characterized. Then, nine main compounds (quercetin, isorhamnetin, kaempferol, ginkgolide A, ginkgolide B, ginkgolide C, bilobalide, tanshinone IIA, and salvianolic acid B) were chosen to further investigate the pharmacokinetic behavior of YDXNT using UPLC-QqQ-MS. The concentration of nine main constituents were in the range of 27.85-76.54 $\mathrm{ng} \mathrm{mL}^{-1}$. This research provides a systematic approach for rapid qualitative analysis of absorbed constituents and for evaluating the pharmacokinetics of the main ingredients of YDXNT following its oral administration. More importantly, this work provides key information on the identification of bioactive compounds and the clarification of their action mechanisms, as well as on the pharmacological actions of YDXNT.

\section{Introduction}

Traditional Chinese medicine (TCM), one of the oldest phytomedicine systems in health care, has been used in Asia, such as in China, Korea, and Japan, for thousands of years. ${ }^{1-4}$ The herbs used in TCM usually have complex formulas and function mainly via their multiple constituents, targets and modes of

\footnotetext{
${ }^{a}$ Institute of Chinese Materia Medica, China Academy of Chinese Medical Science, No. 16 Nanxiaojie, Dongzhimennei Ave., Beijing, 100700, P. R. China. E-mail: liangrixin2009@sina.com; Hongjun0420@sina.com

${ }^{b}$ School of Traditional Chinese Medicine, Capital Medical University, Beijing, 100069, P. R. China

${ }^{c}$ The first clinical medical college of Beijing University of Chinese Medicine, Beijing, 100700, P. R. China

${ }^{d}$ Key Laboratory of Bioactive Substance and Resource Utilisation of the Chinese Herbal Medicine Ministry of Education, Institute of Medicinal Plant Development, Chinese Academy of Medical Sciences, Peking Union Medical College, Beijing, 100193, P. R. China

$\dagger$ Electronic supplementary information (ESI) available. See DOI: 10.1039/c7ra12659j
}

action (3M) in a network-based and holistic manner. ${ }^{5}$ Thus, the therapeutic effects of TCM formulae rely on the joint effect of multiple ingredients. Most TCM formulae are taken orally, and only ingredients absorbed into the blood can exert their bioactivities. ${ }^{6}$ Therefore, it is necessary to trace the compounds in a TCM prescription in vivo and evaluate their pharmacokinetics to provide more in-depth insight into the main active components and therapeutic mechanisms of TCM formulae. In recent years, an integrative strategy using ultra-highperformance liquid chromatography coupled with quadrupole time-of-flight mass spectrometry (UPLC-Q-TOF-MS) combined with ultra-high-performance liquid chromatography with triple quadrupole mass spectrometry (UPLC-QqQ-MS) has been widely used to identify the absorbed constituents and determine main compounds in complex matrices. ${ }^{7-11}$ Hence, they are valuable analytical techniques for identifying key compounds and evaluating the pharmacokinetics of TCM formulae in vivo.

Yindanxinnaotong capsule (YDXNT), a classic TCM formula, comprises eight herbs: Ginkgo biloba leaf (yinxingye, YXY), Salvia miltiorrhizae (danshen, DS1), Gynostemma gynostemmatis 
(jiaogulan, JGL), Erigerontis herba (dengzhanxixin, DZXX), Allii sativi bulbus (dasuan, DS2), Radix/rhizoma notoginseng (sanqi, $\mathrm{SQ}$ ), Crataegi fructus (Shanzha, SZ), and Borneolum (tianranbingpian, TRBP). In China, YDXNT capsule is officially listed in the Chinese Pharmacopoeia 2015 as a prescription for patients suffering from angina pectoris in CHD, hyperlipidemia, stroke, and atherosclerosis. ${ }^{12}$ YDXNT, which has been used to treat cardio-cerebrovascular diseases for many years, ${ }^{13-15}$ is one of the stasis-eliminating formulated products listed in China's National Basic Medical Insurance Drugs Catalogue (http:// www.nhfpc.gov.cn/, 2013 edition).

Our previous research focused on the chemical compounds, quality control, and in vitro and vivo pharmacological properties of YDXNT. In studying the chemical compounds of YDXNT, 70 chemical constituents, including 23 flavonoids, 4 ginkgolides, 15 phenolic compounds, 18 diterpenoid tanshinones, and 10 ginsenosides, were tentatively identified using various detection approaches. Furthermore, fingerprint analysis was established to evaluate the uniform quality of YDXNT soft capsules. ${ }^{16}$ In in vivo experiments, taking YDXNT and swimming may prevent atherosclerosis through a synergistic effect between YDXNT and swimming leading to improved blood circulation, hemorheological parameters, blood lipid levels and vascular endothelium in rats. ${ }^{17}$ In an isolated heart experiment, YDXNT and its main compounds elevated heart function, coronary flow and SOD levels and decreased levels of MDA and inflammatory factors. ${ }^{18}$ Ultimately, our research demonstrated that YDXNT and its major constituents relieve atherosclerosis by regulating lipids, reducing lipid particle deposition in the endothelial layer of arteries, enhancing antioxidant power, and repressing inflammatory activity by inhibiting the nuclear factor-kappa B signaling pathway both in vivo and vitro. ${ }^{19}$ Previous work focus on the pharmacokinetics of main constituents in YXY, DS1, SQ, SZ, and DZXX. ${ }^{20-24}$ However, until now, little has been known about how many constituents are absorbed into the blood after oral administration of YDXNT or about the final fate of the formula in vivo. This presents a huge obstacle to understanding the pharmacological mechanisms of YDXNT. Thus, the present research was designed to determine the absorbed compounds and metabolites in biological samples from rats and investigate the pharmacokinetics of the main abundant active components.

In this research, UPLC-Q-TOF-MS was used to analyze and identify the absorbed ingredients and possible metabolites in rat plasma after oral administration of YDXNT due to its high chromatographic resolution, high sensitivity, short analysis times, good separation, and highly accurate mass values. ${ }^{25-27} \mathrm{In}$ addition, UPLC-QqQ-MS was used for simultaneous quantitation of the main components quercetin (QCT), kaempferol (KMF), isorhamnetin (ISR), ginkgolide A (GA), ginkgolide B (GB), ginkgolide C (GC), bilobalide (BB), tanshinone IIA (TSIIA), and salvianolic acid $\mathrm{B}(\mathrm{SAB})$ in rats, with baicalein used as the internal standard (IS). ${ }^{28,29}$ These compounds exert antiatherosclerotic and endothelium-independent vasodilator effects, prevent inflammatory damage and have a protective effect on nitric oxide, antioxidant action, and anti-vascular inflammation. ${ }^{26-29}$ The pharmacokinetics of QCT, KMF, ISR,
GA, GB, GC, BB, TSIIA, and SAB were investigated in rats after oral administration of YDXNT, and the pharmacokinetics properties of this formula were further speculated. This research represents the first detailed investigation into the absorbed constituents, metabolites, and pharmacokinetics of YDXNT. It may provide valuable information for better understanding the pharmacological mechanisms and clinical applications of YDXNT.

\section{Experiments}

\subsection{Chemicals and reagents}

YDXNT soft capsules were provided by Guizhou Bailing Group Pharmaceutical Co., Ltd. Standards of rutin and cryptotanshinone were purchased from Chengdu Chroma-Biotechnology Co., Ltd. (Sichuan, China). Kaempferol, quercetin, tanshinone IIA, salvianolic acid B, ginkgolide A, ginkgolide B, bilobalide, and baicalein were purchased from Chengdu Herb Purify Co., Ltd. (Sichuan, China). Isorhamnetin, ginkgolide C, ginsenoside Rg1, ginsenoside Re, ginsenoside Rd, and notoginsenoside R1 were purchased from the National Institutes for Food and Drug Control (Beijing, China), Ltd., and HPLC-grade acetonitrile and methanol were obtained from Thermo Fisher Scientific Inc. (Shanghai, China). Formic acid was purchased from Sinopharm Chemical Reagent Co., Ltd. (Shanghai, China), and the water used in the experiments was purified with a Milli-Q system (Sartorius Arium ${ }^{\circledR}$ Pro, Germany).

\subsection{Preparation of YDXNT sample}

The samples were first processed by completely removing the outer capsule layer. Then, a $48.0 \mathrm{~g}$ sample was precisely weighed and soaked with $100 \mathrm{~mL}$ de-ionized water. The sample was ultrasonically dissolved for $30 \mathrm{~min}$ and then stored at $-4{ }^{\circ} \mathrm{C}$ until it was orally administered to rats.

\subsection{Animal experiments}

All animal procedures were performed in accordance with the Guidelines for Care and Use of Laboratory Animals of Center for Laboratory Animal Care, China Academy of Chinese Medical Science and approved by the Animal Ethics Committee of Institute of Chinese Material Medica, China Academy of Chinese Medical Science.

Twenty-four male Sprague-Dawley rats $(250 \pm 30 \mathrm{~g})$ were obtained from the Laboratory Animal Center of the Academy of Military Medical Sciences (Beijing, China) and divided into four groups (six rats each). The rats were housed and fed at a temperature of $25 \pm 2{ }^{\circ} \mathrm{C}$ and a relative humidity of $50 \%$ for three days in the breeding room. Standard chow and deionized water were provided prior to the oral treatment. All rats were fasted for $12 \mathrm{~h}$ with free access to water before the experiment. YDXNT was administered orally to rats at a dose of $4.8 \mathrm{~g} \mathrm{~kg}^{-1}$ $\mathrm{d}^{-1}$, and blood was collected from the abdominal aorta after $20 \%$ urethane anesthesia and then centrifuged at $3000 \mathrm{rpm}$ for 10 min at $4{ }^{\circ} \mathrm{C}$ to obtain the plasma. All samples were stored at $-20{ }^{\circ} \mathrm{C}$ until analysis. Blank plasma samples were prepared in the same manner. 


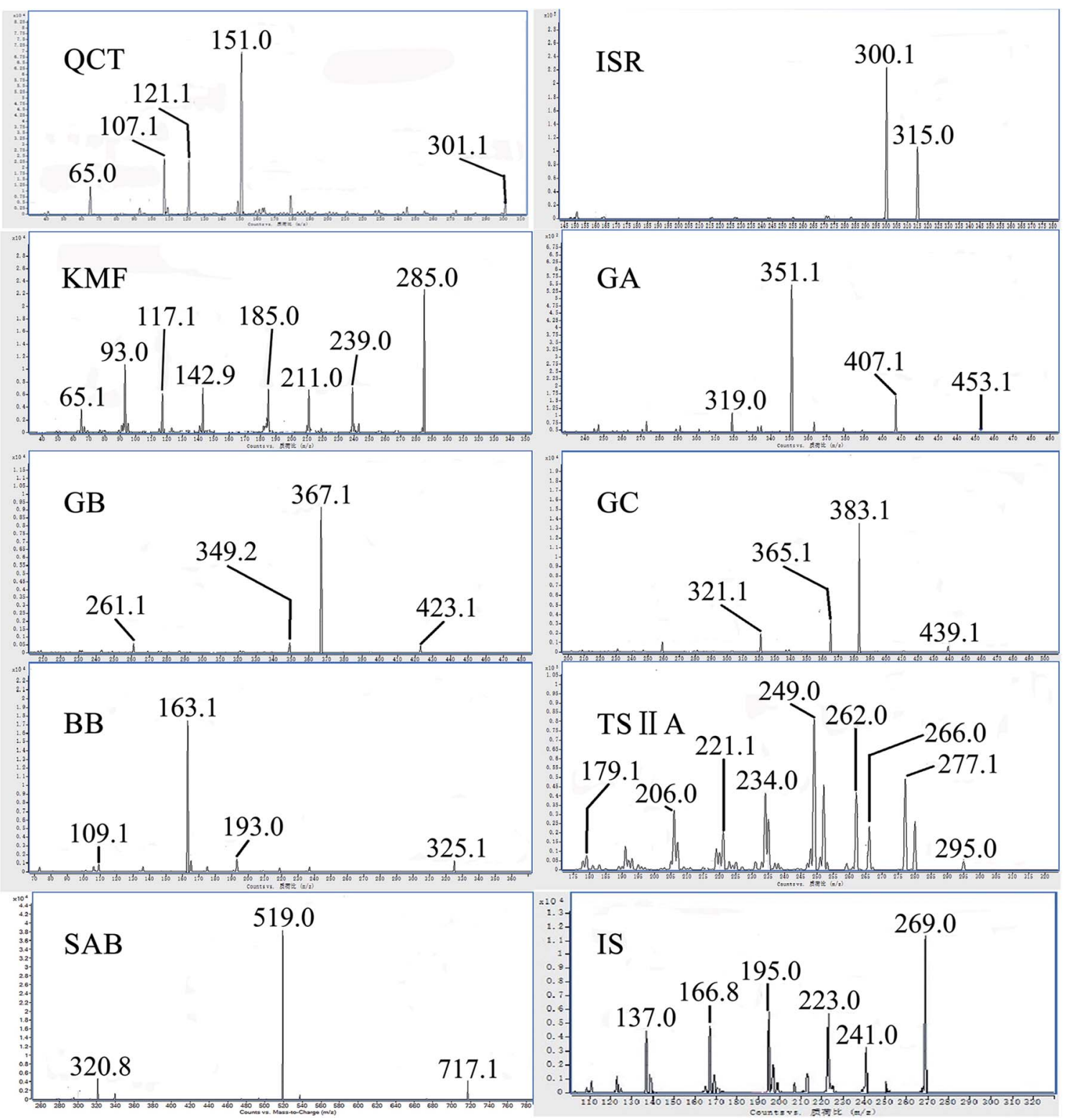

Fig. 1 Product iron mass spectra of the analytes and internal standard (IS). QCT: quercetin; ISR: isorhamnetin; KMF: kaempferol; GA: ginkgolide A; GB: ginkgolide B; GC: ginkgolide C; BB: bilobalide; TSIIA: tanshinone IIA; SAB: salvianolic acid B; IS: baicalein.

\subsection{Serum sample preparation}

Six milliliters of $75 \%$ methanol was added to two milliliters of prepared plasma and then vortexed for $60 \mathrm{~s}$. The mixture was centrifuged at $4000 \mathrm{rpm}$ for $10 \mathrm{~min}$ at $4{ }^{\circ} \mathrm{C}$. The supernatant was transferred to another EP tube and dried using a pressure blowing concentrator. The residue was redissolved with $200 \mu \mathrm{L}$ of $75 \%$ methanol, vortexed for $60 \mathrm{~s}$, and then centrifuged at $13000 \mathrm{rpm}$ for $10 \mathrm{~min}$. To an aliquot of $200 \mu \mathrm{L}$ plasma, $120 \mu \mathrm{L}$ of $0.2 \mathrm{~mol} \mathrm{~L}^{-1}$ acetic acid-sodium acetate buffer solution $(\mathrm{pH}=$ 4.6), $100 \mu \mathrm{L}$ of the IS baicalein and $80 \mu \mathrm{L}$ of $980 \mathrm{U} \mathrm{mL}^{-1} \beta$ glucuronidase and sulfatase was added and then vortexed for $1 \mathrm{~min}$. The mixture was hydrolyzed in a water bath at $37^{\circ} \mathrm{C}$ for $30 \mathrm{~min}$, and $600 \mu \mathrm{L}$ acetone was added to stop the hydrolysis. The sample was cooled, followed by vortexing for $2 \mathrm{~min}$ and then centrifugation at $13000 \mathrm{rpm}$ for $10 \mathrm{~min}$. The supernatant fluid was evaporated to dryness under a flow of nitrogen gas.

\subsection{Preparation of standard, calibration, and quality control solutions}

Stock solutions of QCT, ISR, KMF, GA, GB, GC, BB, TSIIA, SAB and IS were separately prepared by dissolving the compounds in methanol at a concentration of $200 \mathrm{ng} \mathrm{mL}^{-1}$; all solutions were stored at $4{ }^{\circ} \mathrm{C}$. Working solutions for calibration and QC samples were prepared by adding the diluted stock solutions to blank rat plasma. Calibration standards of plasma-derived working solutions with QCT, ISR, KMF, GA, GB, GC, BB, and TSIIA were all prepared with final concentrations ranging from 1-100 $\mathrm{ng} \mathrm{mL} \mathrm{mL}^{-1}$. The SAB solution was prepared with final concentrations ranging 
Table 1 MRM transitions and parameters for the detection of QCT, ISR, KMF, GA, GB, GC, BB, TSIIA, SAB and IS

\begin{tabular}{lllll}
\hline Analyte & MRM transitions & Fragementor $(\mathrm{V})$ & Collision energy $(\mathrm{V})$ & Dwell time $(\mathrm{ms})$ \\
\hline QCT & $301.1 \rightarrow 151.0$ & 350 & 26 & 20 \\
ISR & $315.0 \rightarrow 300.1$ & 350 & 22 & 20 \\
KMF & $285.0 \rightarrow 142.9$ & 350 & 35 & 20 \\
GA & $453.1 \rightarrow 351.1$ & 350 & 16 & 20 \\
GB & $423.1 \rightarrow 367.1$ & 350 & 15 & 20 \\
GC & $439.1 \rightarrow 383.1$ & 350 & 12 & 20 \\
BB & $325.1 \rightarrow 163.1$ & 350 & 26 & 20 \\
TSIIA & $295.0 \rightarrow 249.0$ & 350 & 38 & 20 \\
SAB & $717.1 \rightarrow 519.0$ & 350 & 28 & 20 \\
IS & $269.0 \rightarrow 195.0$ & & &
\end{tabular}

from 5-500 $\mathrm{ng} \mathrm{mL}^{-1}$. The IS solution was prepared at a final concentration of $100 \mathrm{ng} \mathrm{mL} \mathrm{m}^{-1}$ in methanol. For method validation, QC plasma samples of QCT, ISR, KMF, GA, GB, GC, BB, and TSIIA were all prepared separately at three concentrations $(1,10$ and $100 \mathrm{ng} \mathrm{mL}^{-1}$ ). The QC plasma sample of SAB was prepared at three concentrations $\left(5,50\right.$, and $\left.500 \mathrm{ng} \mathrm{mL}^{-1}\right)$.

\subsection{UPLC-Q-TOF-MS for qualitative analysis}

UPLC data were produced using the Waters Synapt G2-S UPLC system (Waters, Milford, USA) equipped with a quaternary pump system, online degasser, autosampler, thermostatically controlled column compartment, and diode array detector. The system was controlled with Masslynx V4.1 software. The chromatographic column ACQUITY BEH C18 $(100 \times 2.1 \mathrm{~mm}, 1.7$ $\mu \mathrm{m})$ equipped with VanGuard ${ }^{\mathrm{TM}}$ BEHC18 $1.7 \mu \mathrm{m}$ was used. The column was eluted with a gradient of $\mathrm{A}(0.1 \%$ formic acid in deionized water) and $\mathrm{B}$ (CAN) at a flow rate of $0.3 \mathrm{~mL} \mathrm{~min}^{-1}$ and a temperature of $30{ }^{\circ} \mathrm{C}$ : 0-2.5 $\mathrm{min}, 10-16 \% \mathrm{~B} ; 2.5-6.5 \mathrm{~min}, 16-$ 16\% B; 6.5-9.5 min, 16-25\% B; 9.5-11.0 min, 25-25\% B; 11.0$12.0 \mathrm{~min}, 25-45 \% \mathrm{~B} ; \mathbf{1 2 . 0}-15.0 \mathrm{~min}, 45-55 \% \mathrm{~B}$; 15.0-18.0, 55-
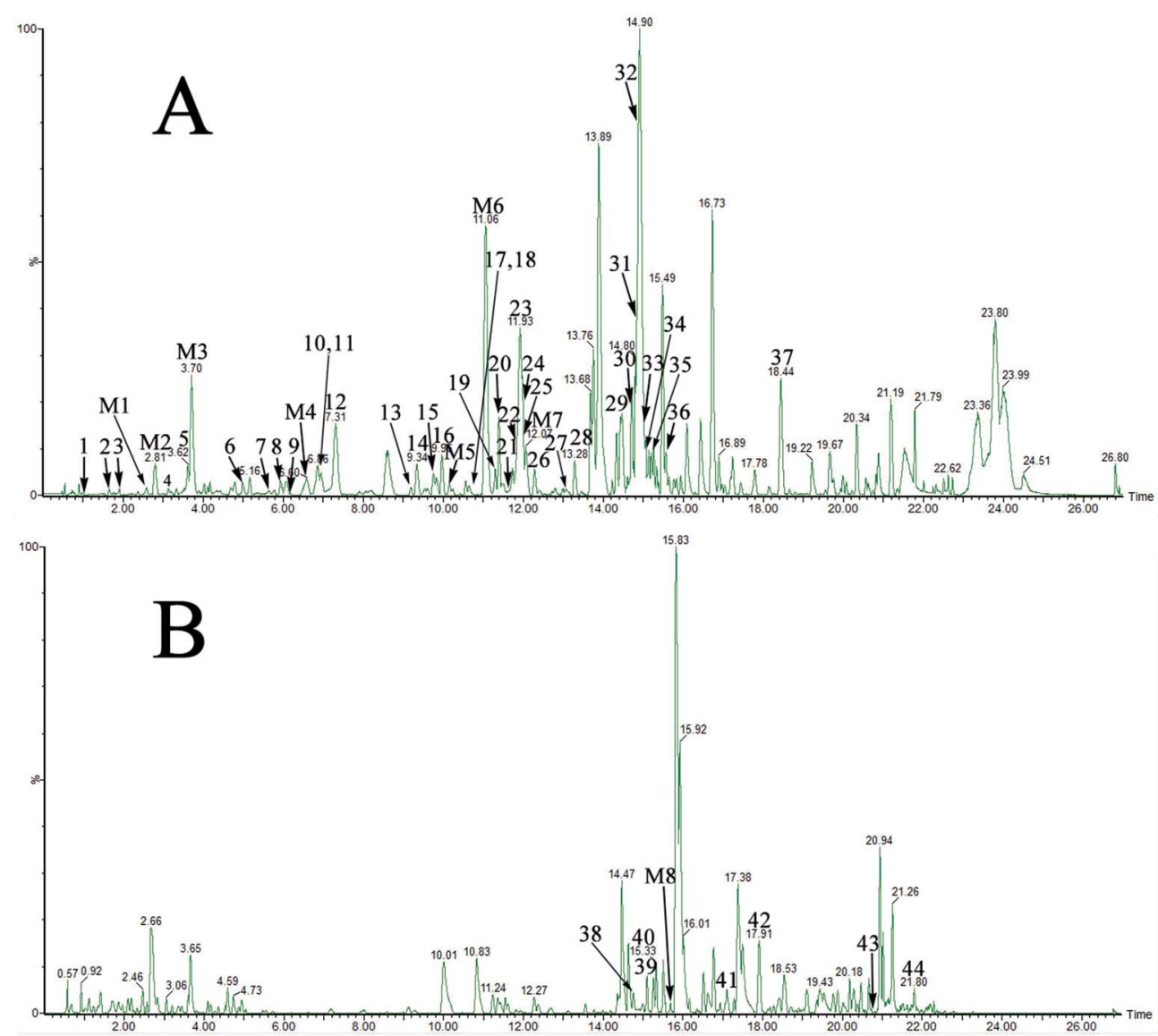

Fig. 2 The UPLC-Q-TOF-MS results for drug-containing plasma. (A) negative ionisation mode-analysis of drug-containing plasma. (B) positive ionisation-mode analysis of drug-containing plasma. 


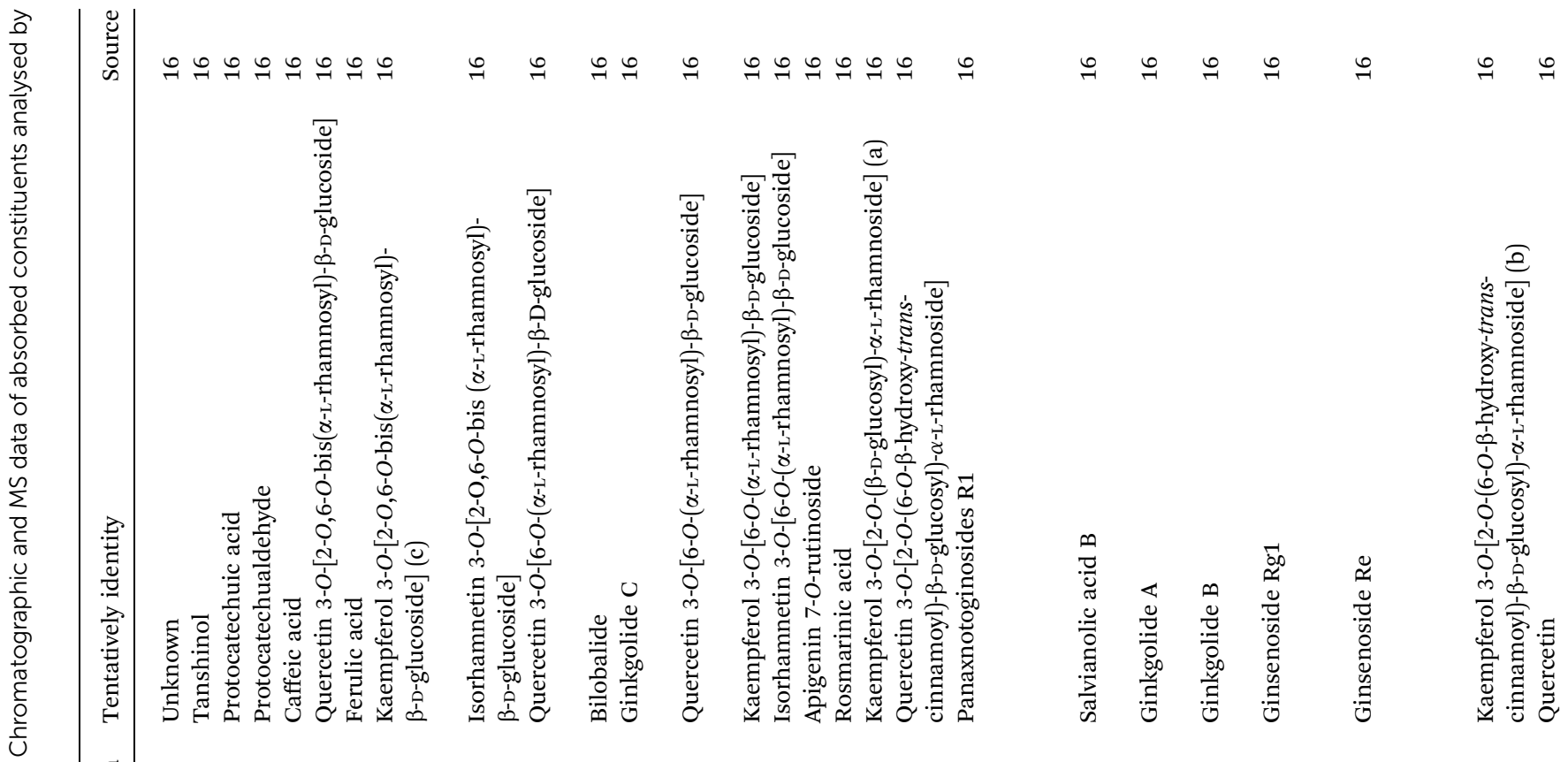

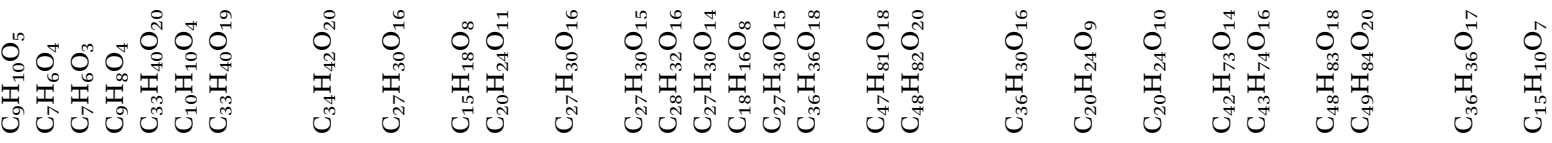

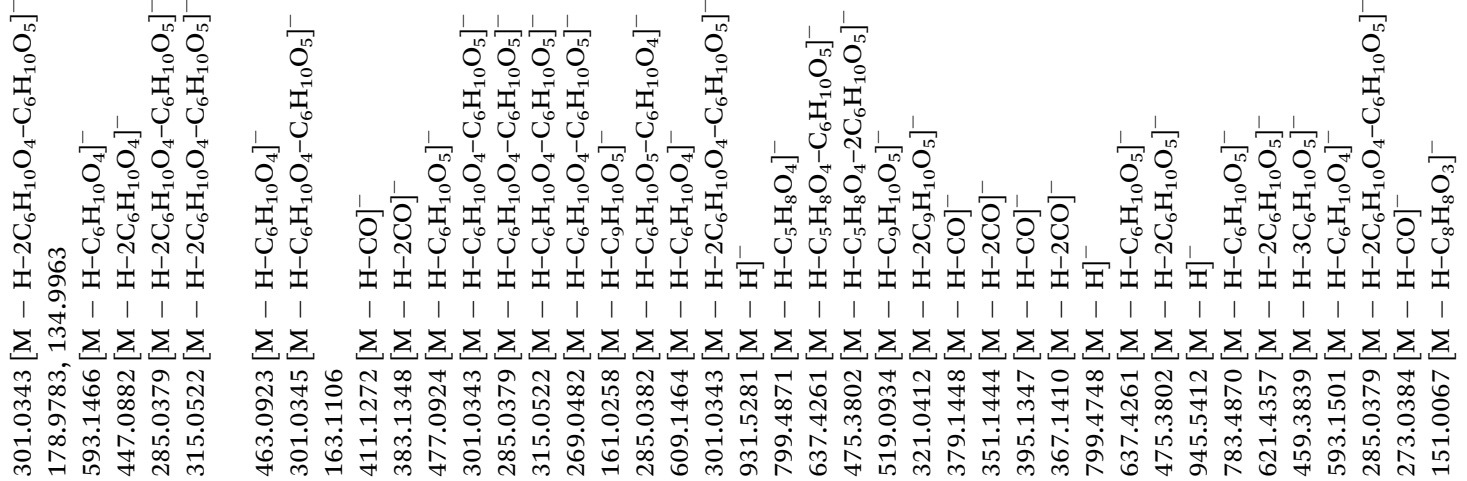

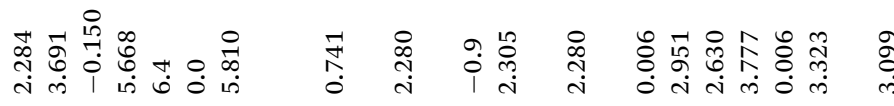

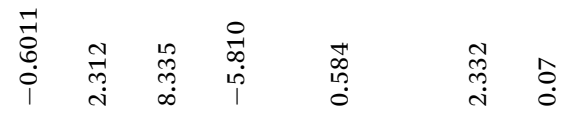




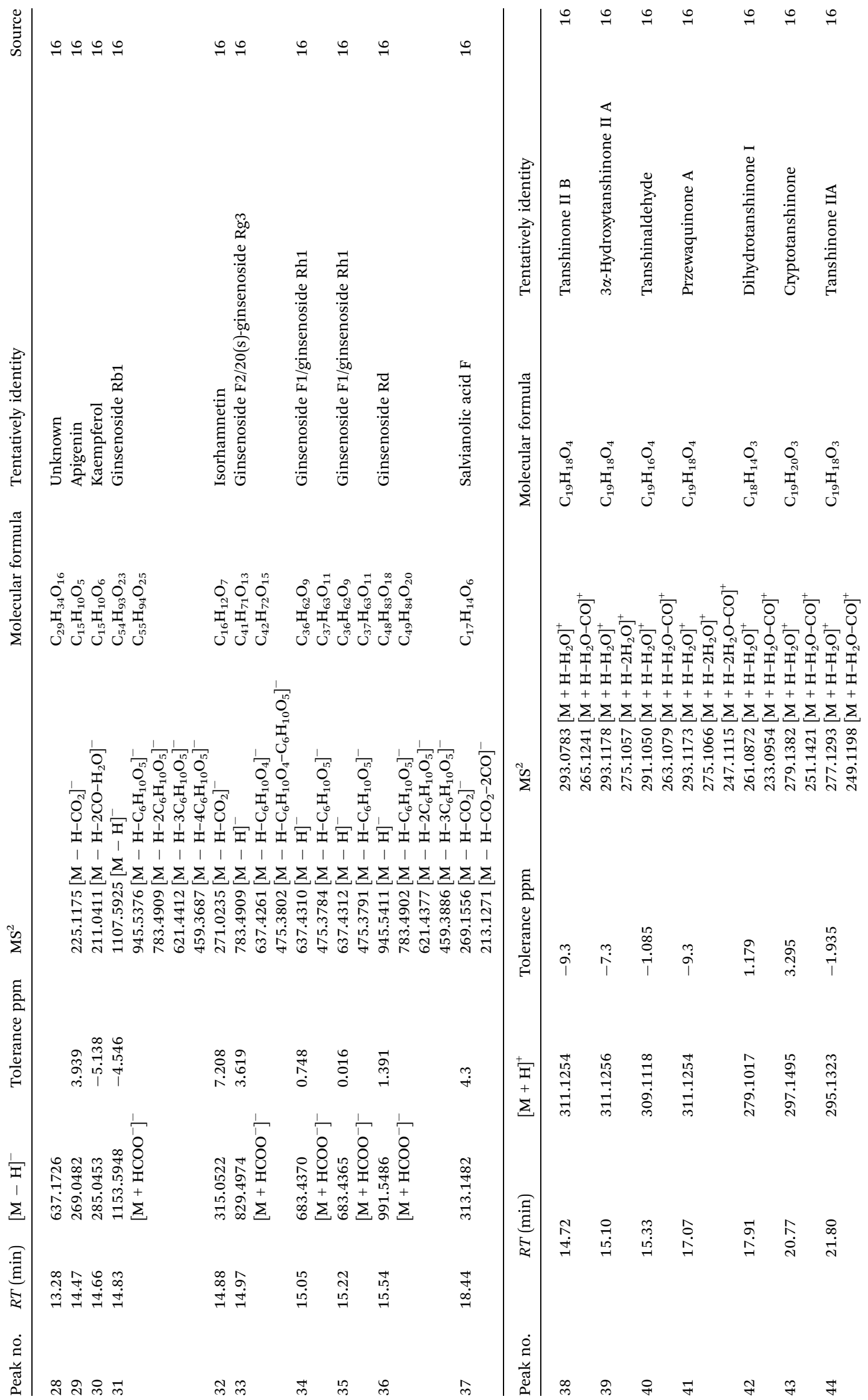




(1)

政

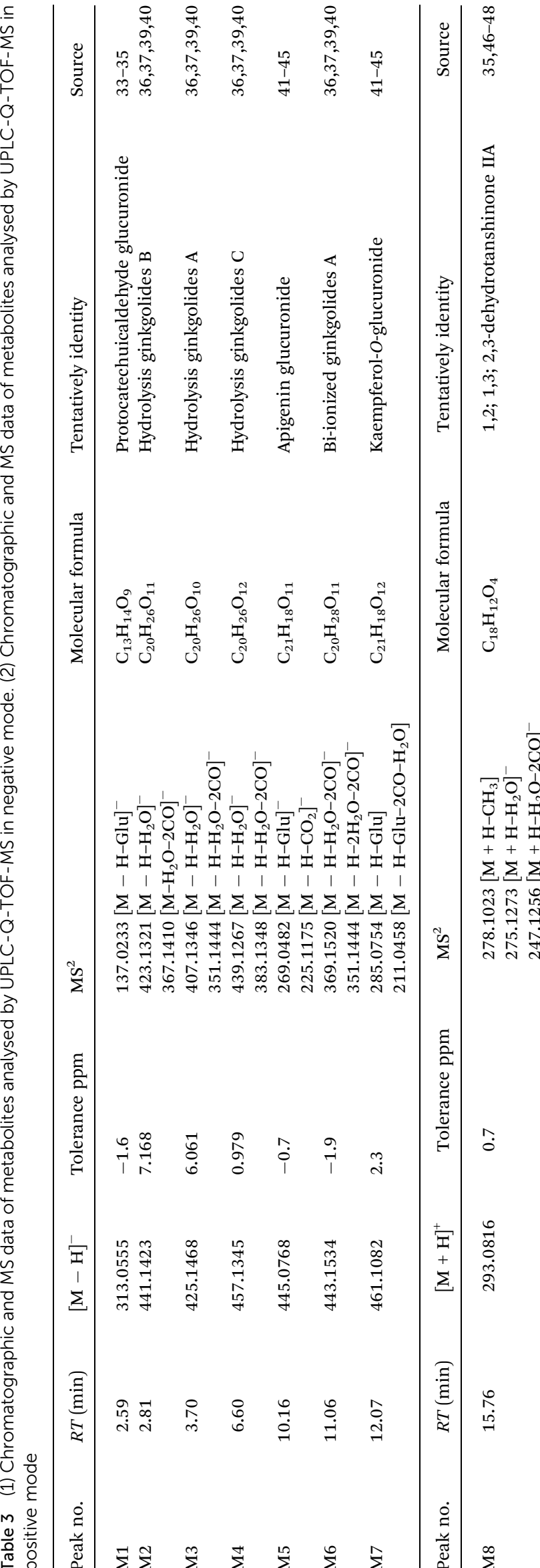

98\% B; 18.0-21.0 min, 98-98\% B; $21.0-25.0$ min, $98-10 \%$ B. An injection volume of $10 \mu \mathrm{L}$ was used for analysis.

For mass detection, mass data were recorded using the Waters Synapt G2-S Q-TOF Mass Spectrometer system equipped with an atmospheric pressure chemical ionization (APCI) electrospray ionization (ESI) interface (Waters, Milford, USA). The conditions of the TOF/MS were set as follows: capillary, $2.5 \mathrm{kV}$; source temperature, $120{ }^{\circ} \mathrm{C}$; cone gas flow, $50 \mathrm{~L} \mathrm{~h}^{-1}$; nebulizer, 6 bar; desolvation temperature, $450{ }^{\circ} \mathrm{C}$; sheath gas flow, 12 L $\min ^{-1}$; nozzle voltage, 10-1000 V; The mass spectrometer was operated from 100 to $1700 \mathrm{~m} / \mathrm{z}$. Accurate masses and compositions of the precursor and fragment ions were calculated using MassLynx 4.1 software. The UPLC and Q-TOF-MS methods have been optimised (ESI $1 \dagger$ ).

\subsection{UPLC-QqQ-MS for quantitative analysis}

Chromatographic analysis was performed using an Agilent 1290 Series UPLC from Agilent Technologies (Palo Alto, CA, USA), equipped with a quaternary pump an online degasser, autosampler and column oven. Chromatographic separation was performed on the Agilent ZORBAX SB-Aq C18 column $(100 \mathrm{~mm}$ $\times 2.1 \mathrm{~mm}, 1.8 \mu \mathrm{m}$, Agilent, USA). The analytical column temperature was maintained at $30{ }^{\circ} \mathrm{C}$ and eluted with a mobile phase comprising $0.1 \%$ formic acid in water (A) and acetonitrile (B) using the following gradient program: $25-90 \%$ B from 0 to $1 \mathrm{~min}, 90 \% \mathrm{~B}$ from 1 to $3.5 \mathrm{~min}$, and $90-25 \% \mathrm{~B}$ from 3.5 to $5 \mathrm{~min}$ at a flow rate of $0.3 \mathrm{~mL} \mathrm{~min}^{-1}$. The equilibration time was $5 \mathrm{~min}$, and the injection volume of sample and reference constituents was $5 \mu \mathrm{L}$.

Mass spectrometry was performed on the Agilent 6490 triplequadrupole mass spectrometer (Palo Alto, CA, USA) equipped with an atmospheric pressure chemical ionization (APCI) interface. The mass spectrometer was set in negative ionization mode, with the capillary voltage set at $3500 \mathrm{~V}$. The other parameters in the source were set as follows: desolvation gas flow, $15 \mathrm{~L} \min ^{-1}$; source temperature, $290{ }^{\circ} \mathrm{C} ; \mathrm{N}_{2}$ sheath gas temperature, $350{ }^{\circ} \mathrm{C}$; nebulizer gas $\left(\mathrm{N}_{2}\right)$ pressure, $50 \mathrm{psi}$; flow, 12 $\mathrm{L} \min ^{-1}$. The mass spectrometer scanned in multiple reaction monitoring (MRM) mode. The UPLC and QqQ-MS methods have been optimised (ESI $2 \dagger$ ). Fig. 1 shows the MS/MS product ion spectra of the analytes and IS. The optimized extraction ion pairs and parameter values are shown in Table 1.

\subsection{Method validation}

Method validation was performed according to the guidelines for bioanalytical method validation published by the US FDA. ${ }^{30}$ The analytical method used in this research was validated to demonstrate its specificity, selectivity, linearity, lower limit of quantification (LLOQ), precision, accuracy, stability, extraction recovery rates and matrix effects of the samples (ESI $3 \dagger$ ).

\subsection{Pharmacokinetics study}

Six male rats were used in the pharmacokinetics study. All the rats were orally administered $2.4 \mathrm{~g} \mathrm{~kg}^{-1}$ YXCNT suspended in an aqueous solution of $0.5 \%$ carboxymethyl cellulose sodium (QCT: 4.118 mg; KMF: 5.067 mg; ISR: $0.965 \mathrm{mg}$; GA: 1.416 mg; 


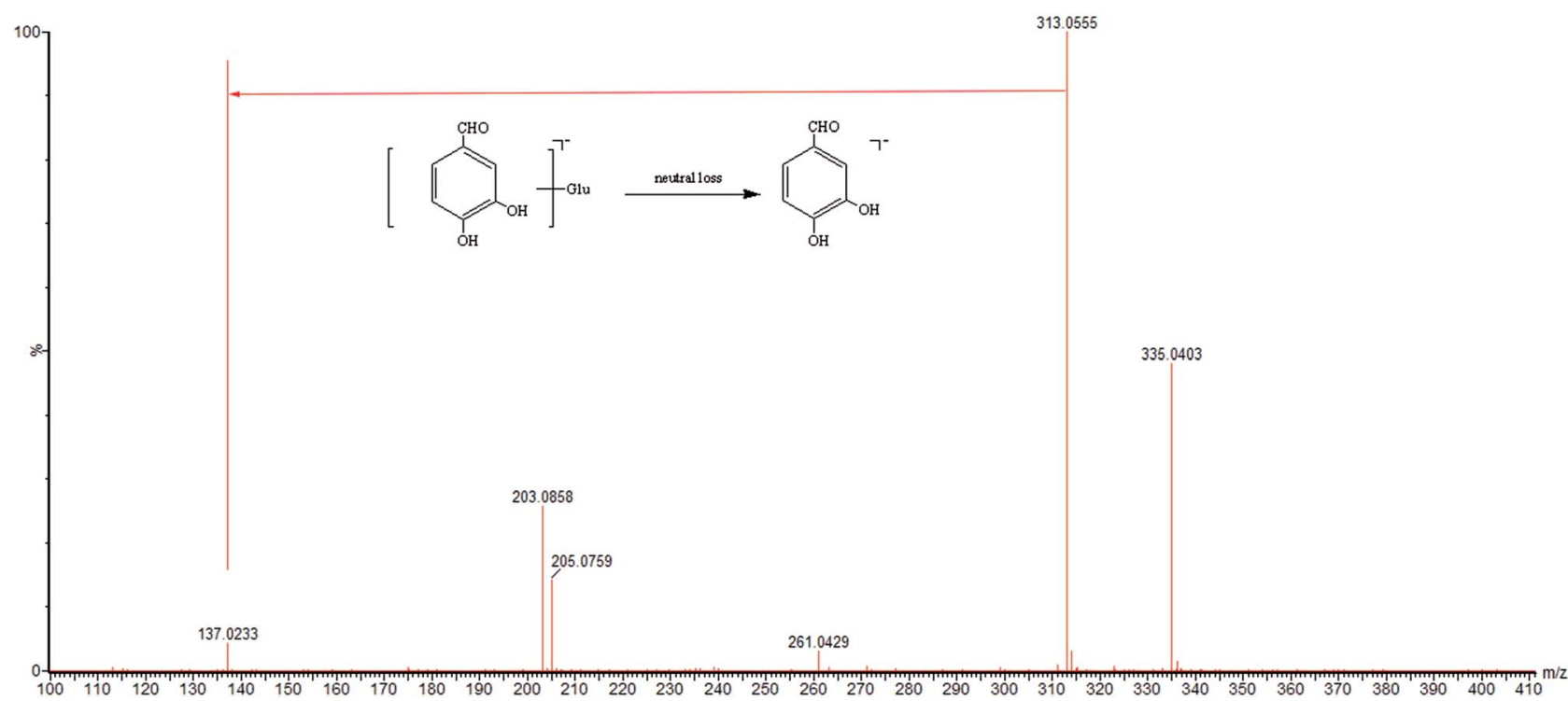

Fig. 3 The mass spectra of protocatechuic aldehyde glucuronide in negative mode and the proposed fragmentation pathway.

GB: $0.680 \mathrm{mg}$; GC: $0.796 \mathrm{mg}$; BB: $0.326 \mathrm{mg}$; TSIIA: $0.624 \mathrm{mg}$; SAB: $3.84 \mathrm{mg}$ ). After dosing for $0 \mathrm{~min}, 5 \mathrm{~min}, 15 \mathrm{~min}, 20 \mathrm{~min}$, $30 \mathrm{~min}, 45 \mathrm{~min}, 1 \mathrm{~h}, 1.5 \mathrm{~h}, 2 \mathrm{~h}, 3 \mathrm{~h}, 4 \mathrm{~h}, 6 \mathrm{~h}, 8 \mathrm{~h}, 12 \mathrm{~h}$, and $24 \mathrm{~h}$, blood samples $(500 \mu \mathrm{L})$ were drawn from the retro-orbital plexus of each mouse and collected in heparinized tubes according to the specific schedule. All the collected blood samples were immediately centrifuged at $13000 \mathrm{rpm}$ for $10 \mathrm{~min}$. The plasma layer was transferred to clean tubes and stored at $-20{ }^{\circ} \mathrm{C}$ until analysis. The plasma concentrations of QCT, ISR, KMF, GA, GB, GC, BB, TSIIA, and SAB were expressed as the mean \pm standard deviation (SD), and the mean concentration-time curve was plotted. Non- compartmental pharmacokinetic

parameters

were

\section{Results and discussion}

\subsection{Identification of prototype constituents and metabolites} in drug-containing plasma

The optimized LC-MS protocol was used for the separation and identification of compounds in drug-containing plasma. Exogenous constituents of YDXNT in the plasma were divided into prototype components and metabolites. Ultimately, a total of 52 compounds, including 44 prototype constituents and 8

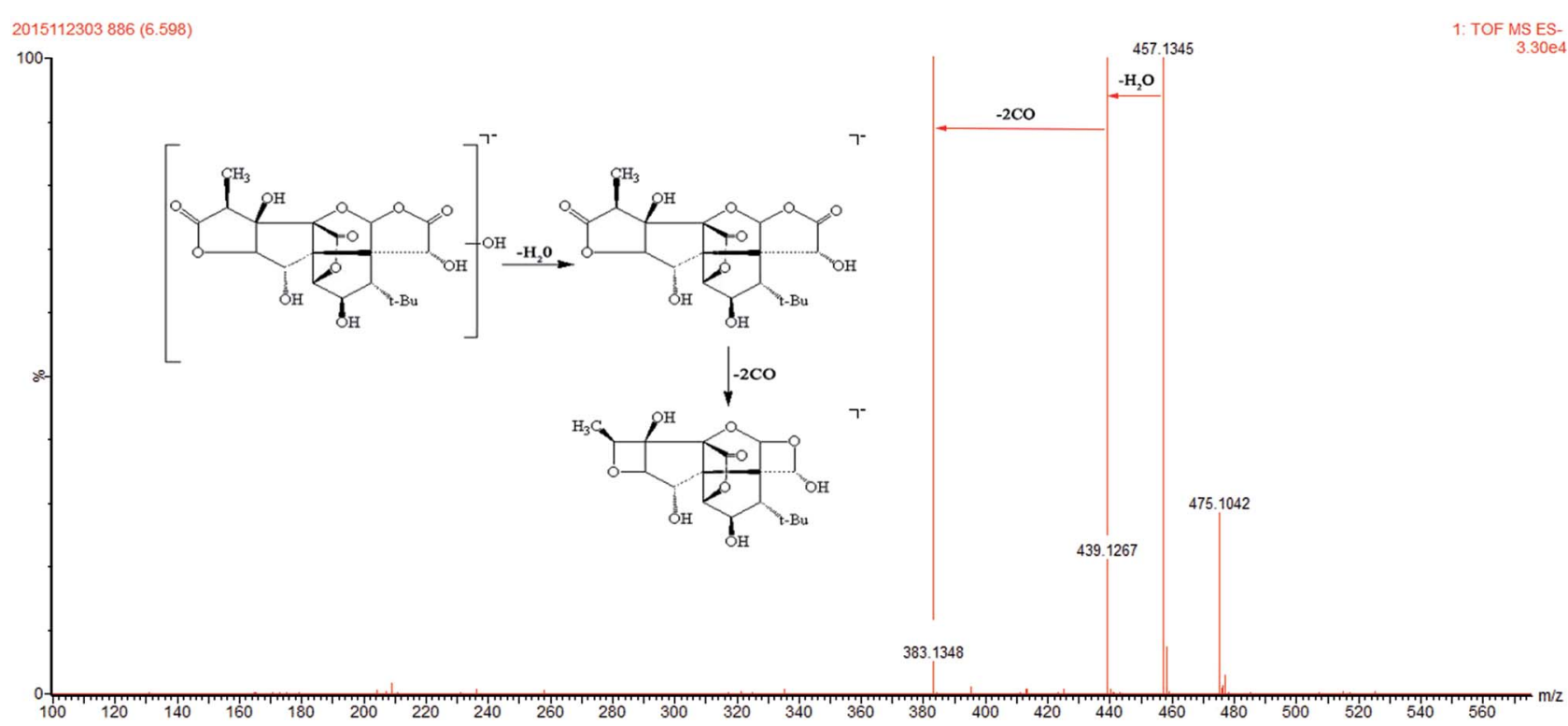

Fig. 4 The mass spectra of hydrolysis ginkgolides $C$ in negative mode and the proposed fragmentation pathway. 


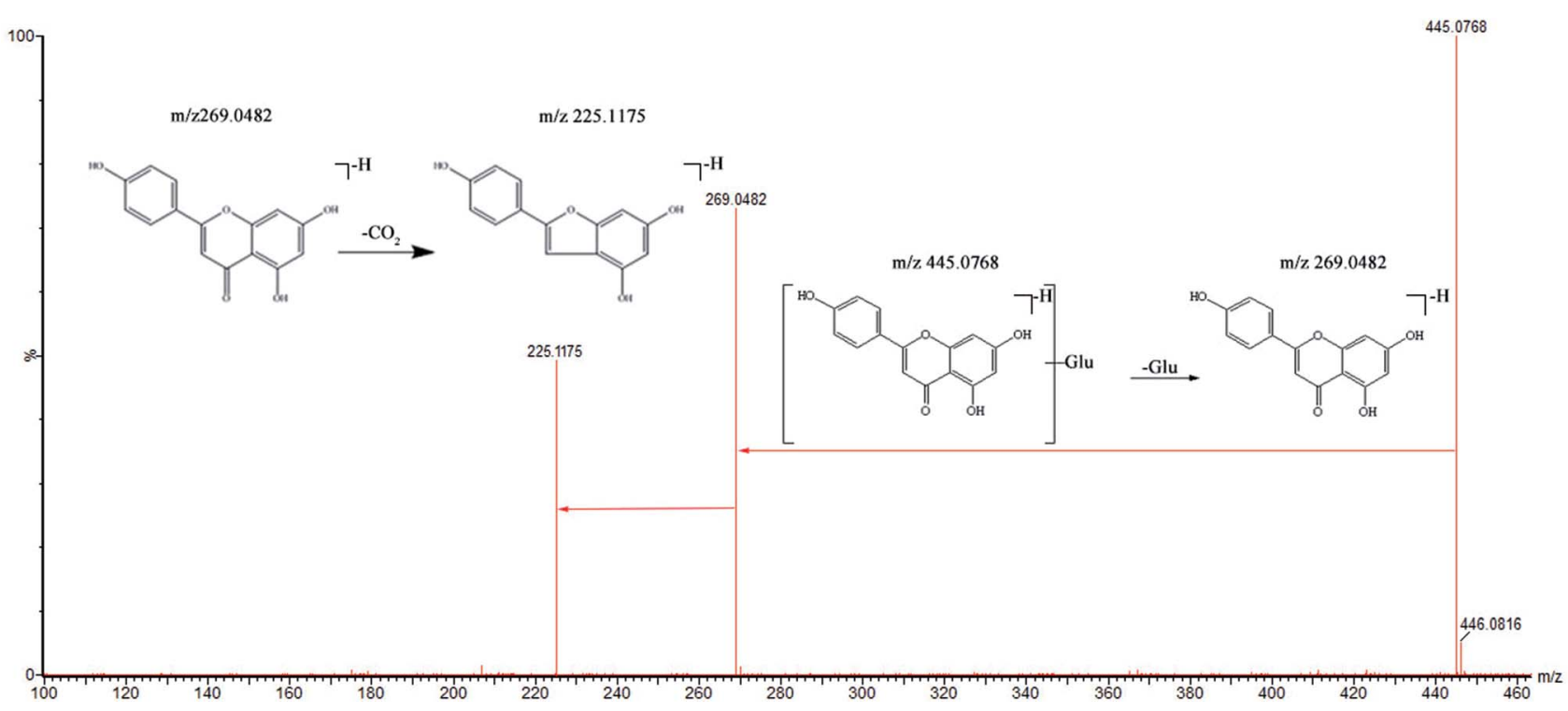

Fig. 5 The mass spectra of apigenin glucuronide in negative mode and the proposed fragmentation pathway.

metabolites, were detected by comparison to the blank plasma sample (Fig. 2, Tables 2 and 3).

\subsection{Analysis of prototype constituents of YDXNT in rat plasma}

Owning to the high sensitivity of UPLC-Q-TOF-MS and extracted ion chromatograms, 44 prototype constituents were tentatively identified by comparison with standards, mass data, fragment information, and our previous compound research. ${ }^{16}$ Of the 44 peaks, 36 were detected in negative-ionization mode, and 7 peaks were detected in positive-ionization mode. The peaks included 15 flavonoids, 3 ginkgolide, 8 phenolic, 8 ginsenosides, and 7 diterpenoid tanshinones. Among them, 14 peaks were further confirmed with standards by comparing retention time and MS data, including peaks 10, 11, 12, 20, 21, $22,23,24,26,27,30,32,36,43$, and 44 .

\subsection{Analysis of the metabolites of YDXNT in rat plasma}

The full-scan mass spectrum was achieved from the plasma sample after YDXNT was orally administered to rats and compared to the blank plasma sample to discover possible metabolites in rat plasma. Eight metabolites, including 1 monophenol, 4 ginkgolides, 2 flavonoids, and 1 diterpenoid tanshinone, were tentatively identified from the plasma by

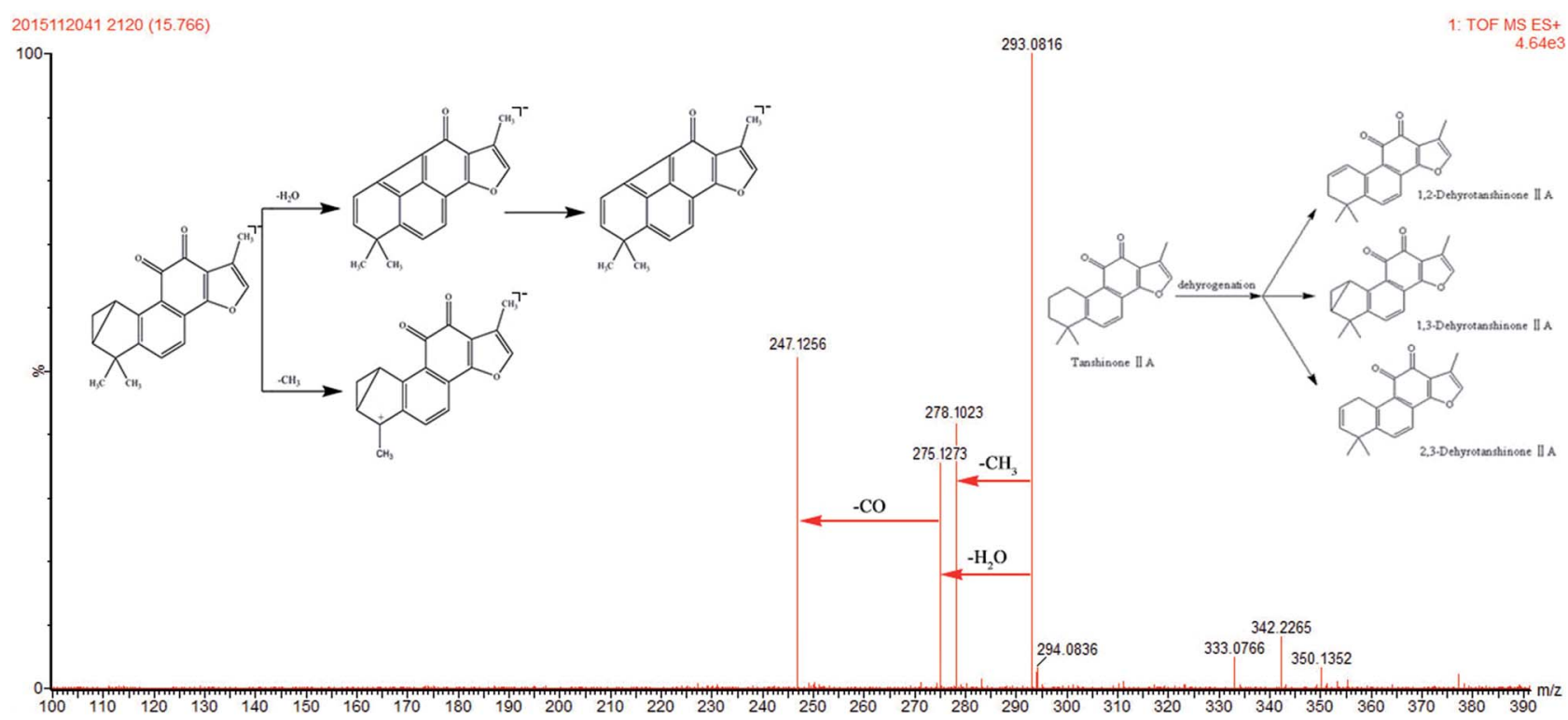

Fig. 6 The mass spectra of 1,2; 1,3; 2,3-dehydrotanshinone IIA in positive mode and the proposed fragmentation pathway. 
Table 4 Regression data and LLOQs of BB, GA, GB, GC, QCT, KMF, and ISR

\begin{tabular}{|c|c|c|c|c|c|}
\hline Biosamples & Analyte & $\begin{array}{l}\text { Linear range } \\
\left(\mathrm{ng} \mathrm{mL}^{-1}\right)\end{array}$ & Linear equation & $R^{2}$ & LLOQ (ng mL $\mathrm{mL}^{-1}$ ) \\
\hline \multirow[t]{6}{*}{ Plasma } & QCT & $1-100$ & $Y=0.0443 X+0.0621$ & 0.9993 & 1 \\
\hline & KMF & $1-100$ & $Y=0.0031 X+0.0222$ & 0.9971 & 1 \\
\hline & GA & $1-100$ & $Y=0.00741 X+0.3012$ & 0.9987 & 1 \\
\hline & GB & $1-100$ & $Y=0.1355 X+1.3265$ & 0.9987 & 1 \\
\hline & TSIIA & $1-100$ & $Y=0.3066 X+0.3920$ & 0.9992 & 1 \\
\hline & $\mathrm{SAB}$ & $5-500$ & $Y=0.0063 X+0.0542$ & 0.9985 & 5 \\
\hline
\end{tabular}

comparing their retention times, deprotonated molecules, and characteristic fragments to those reported in the literature..$^{31-47}$ The precursors of these metabolites were deduced to be protocatechuic aldehyde, ginkgolide A, ginkgolide B, ginkgolide C, apigenin, kaempferol, and tanshinone IIA. Characterization of the structures of these metabolites is described below.

In our research, one monophenolic-related metabolite (M1) was detected. M1 $\left(t_{\mathrm{R}}=2.59 \mathrm{~min}\right)$ showed a deprotonated molecular ion at $m / z 313.0555$, and product ions of $m / z 137.0233$ were obtained. The neutral loss of $176 \mathrm{Da}\left(\mathrm{C}_{6} \mathrm{H}_{8} \mathrm{O}_{6}\right)$ indicated that M1 was the glucuronide conjugate ${ }^{32,33}$ (Fig. 3). Based on retention time, accurate quasi-molecular ion, and MS/MS data, as well as the related literature, ${ }^{32-34} \mathrm{M} 1$ was tentatively identified as the characteristic ion of protocatechuic aldehyde glucuronide.

Ginkgolide-related metabolites were the main possible xenobiotic metabolites in plasma. ${ }^{35,36}$ In the present study, 3 ginkgolides and 4 ginkgolide-related metabolites were detected. The 3 ginkgolides (ginkgolide $\mathrm{A}$, ginkgolide $\mathrm{C}$ and ginkgolide $\mathrm{B}$ ) were identified by comparison to standards. The characteristic ions at $\mathrm{m} / \mathrm{z} 351, \mathrm{~m} / \mathrm{z} 383$ and $\mathrm{m} / \mathrm{z} 367$ were observed in ginkgolide A, ginkgolide $\mathrm{C}$, and ginkgolide $\mathrm{B}$, respectively. The common fragmentation pattern of ginkgolide was the successive loss of $2 \mathrm{CO}^{37} \mathrm{M} 2, \mathrm{M} 3$ and $\mathrm{M} 4$ have $[\mathrm{M}-\mathrm{H}]^{-}$ions at $\mathrm{m} / \mathrm{z}$

Table 5 Precision and accuracy for bioactive compounds in rat plasma and brain homogenates ( $n=18,6$ replicates per day for 3 days)

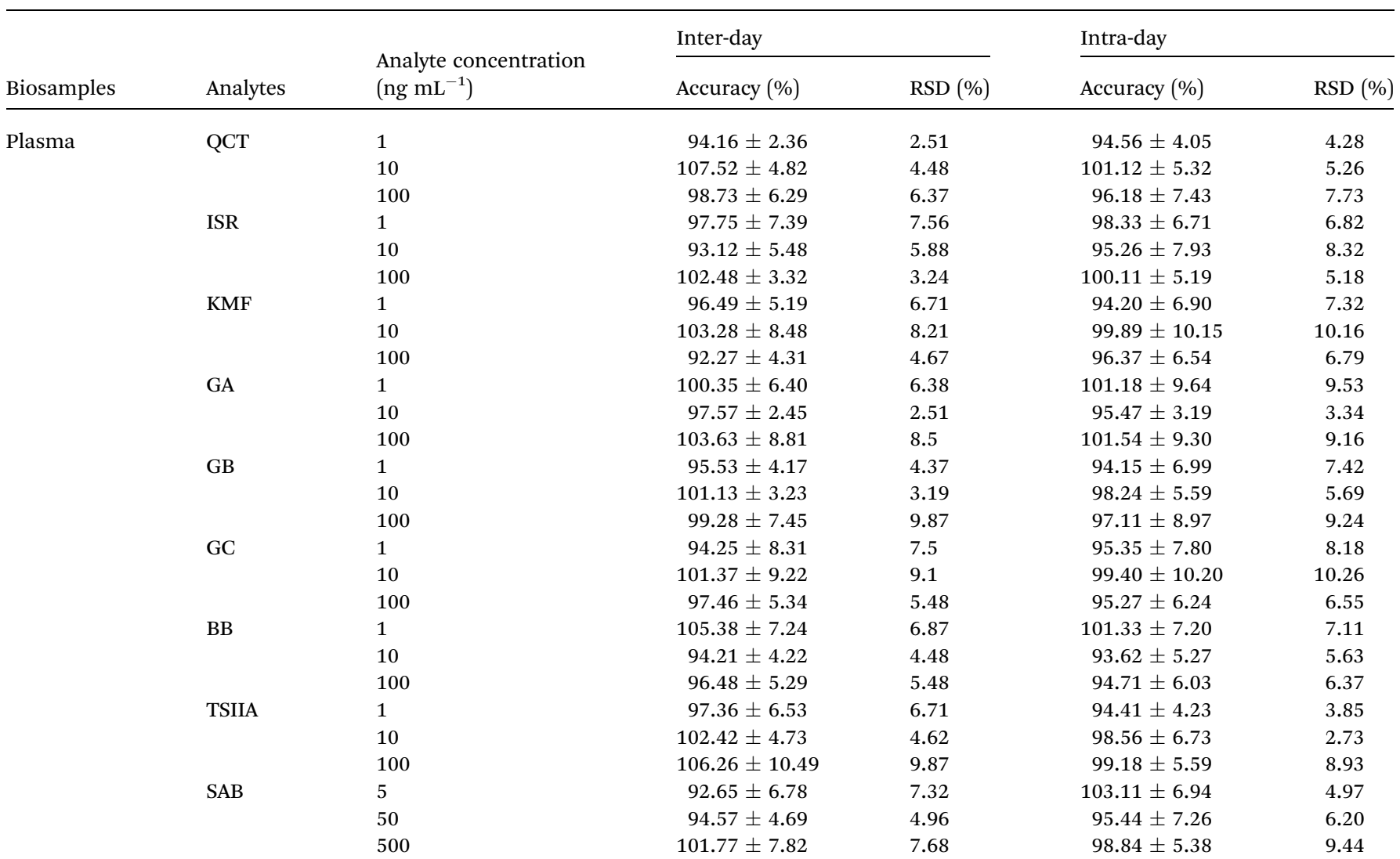


Table 6 Extraction recovery and matrix effect for the analytes or bioactive compounds in rat plasma and brain homogenates $(n=6)$

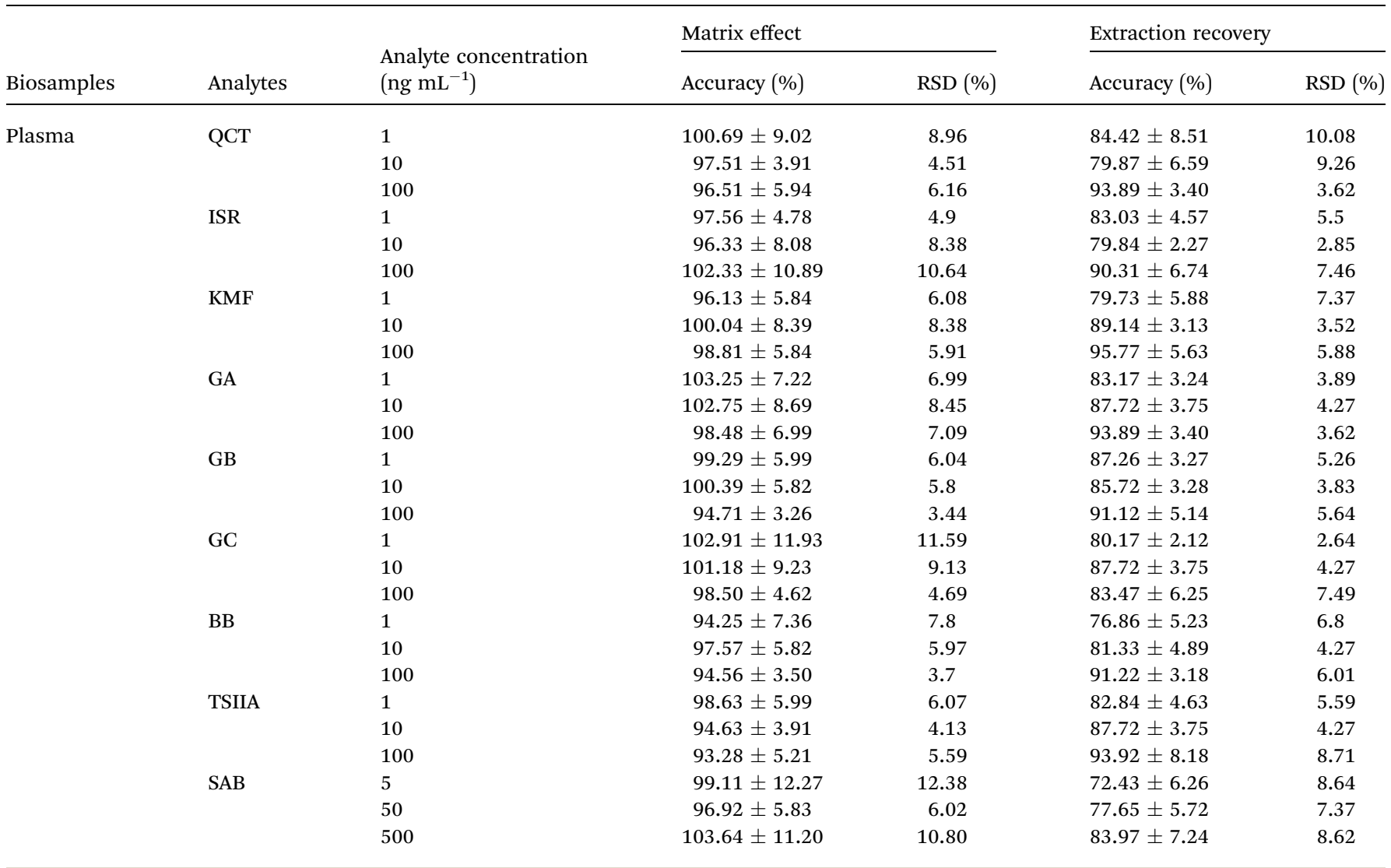

441.1423, $m / z$ 425.1468, and $m / z 457.1345$, which were 18 Da heavier than ginkgolide $\mathrm{B}$, ginkgolide $\mathrm{A}$, and ginkgolide $\mathrm{C}$, respectively. The main fragment ions of M2, M3, and M4 in the MS/MS spectra were $367.1410,351.1444$, and 383.1348 by the loss of $74 \mathrm{Da}$. Three metabolites both exhibited fragment ions of $\left[\mathrm{M}-\mathrm{H}-\mathrm{H}_{2} \mathrm{O}-2 \mathrm{CO}\right]$, which have a similar mass schizolysis rule as seen for ginkgolide. According to the above analysis, we tentatively presumed that M2 is a hydrolyzed metabolite of ginkgolide B, M3 is a hydrolyzed metabolite of ginkgolide A, and M4 is a hydrolyzed metabolite of ginkgolide C (Fig. 4)..$^{35,36,38,39}$ Furthermore, M6 ( $\mathrm{m} / \mathrm{z} 443.1534)$ was tentatively identified as another hydrolyzed metabolite of ginkgolide A (bi-ionized ginkgolide A) by comparing deprotonated molecular $[\mathrm{M}-\mathrm{H}]^{-}$ and characteristic fragments $[\mathrm{M}-\mathrm{H}-74]$ to those reported in the literature. ${ }^{36}$

Two flavonoid-related metabolites were detected. M5 $\left(t_{\mathrm{R}}=\right.$ 10.16) and $\mathrm{M} 7\left(t_{\mathrm{R}}=12.05\right)$ showed quasi-molecular ions at $\mathrm{m} / \mathrm{z}$ 445.0768 and $m / z 461.1082$, respectively, in the MS spectra. Two metabolites showed product ions at $\mathrm{m} / \mathrm{z} 269.0482$ and $\mathrm{m} / \mathrm{z}$ 285.0754 in the MS/MS spectra. The loss of $176 \mathrm{Da}$ indicated that M5 and M7 were glucuronide conjugates. Alternatively, loss of $\mathrm{CO}_{2}$ from the product ion $(m / z 269.0482)[\mathrm{M}-\mathrm{H}-176-44]^{-}$ and loss of $2 \mathrm{CO}$ and $\mathrm{H}_{2} \mathrm{O}$ from the product ion $(\mathrm{m} / \mathrm{z} 285.0754)$ $[\mathrm{M}-\mathrm{H}-176-74]^{-}$occurred. The pathway of further fragmentation was similar to that of apigenin and kaempferol; ${ }^{16}$ therefore, M5 and M7 were tentatively identified as apigenin- $O$ - glucuronide (Fig. 5) and kaempferol-O-glucuronide by comparing retention times, accurate quasi-molecular ions, and MS/MS fragmentation data as well as by referring to the related literature. ${ }^{\mathbf{4 0 - 4 4}}$

One tanshinone-related metabolite was tentatively identified from rat plasma on the basis of accurate deprotonated molecular data, MS/MS fragmentation data, and previous reports. ${ }^{45-47}$ $\mathrm{M} 7$ gives rise to a protonated molecule $[\mathrm{M}+\mathrm{H}]^{+}$at $\mathrm{m} / z$ 293.0816. This molecule was 2 Da smaller than TSIIA. The neutral loss of $m / z 15, m / z 18$, and $m / z 28$ indicated the loss of $\mathrm{CH}_{3}, \mathrm{H}_{2} \mathrm{O}$, and $\mathrm{CO}$, respectively (Fig. 6). Corresponding to the deprotonated molecular ion and its fragments, M7 was tentatively identified as dehydrogenated TSIIA, which has been reported in the literature. ${ }^{34,45-47}$

\subsection{Assay validation}

3.4.1 Specificity. Under the UPLC-MS/MS conditions, a specific voltage at the optimal value was set to obtain the best sensitivity and specificity for each analyte. According to the results, there was no obvious interference from endogenous components, indicating good method selectivity. The chromatographic peaks were at retention times of $2.435,2.580$, $2.557,2.499,2.494,2.324,2.454,3.384,2.285$ and $2.612 \mathrm{~min}$ for QCT, ISR, KMF, GA, GB, GC, BB, TSIIA, SAB and IS, respectively.

3.4.2 Linearity. The calibration curves showed good linearity $\left(R^{2}>0.9931\right)$ over the concentration ranges of the nine 
Table 7 Stability of the analytes or bioactive compounds in rat plasma and brain homogenates $(n=6)$

\begin{tabular}{|c|c|c|c|c|c|c|c|c|c|c|}
\hline \multirow[b]{2}{*}{ Biosamples } & \multirow[b]{2}{*}{ Analytes } & \multirow{2}{*}{$\begin{array}{l}\text { Analyte } \\
\text { concentration } \\
\left(\mathrm{ng} \mathrm{mL}^{-1}\right)\end{array}$} & \multicolumn{2}{|c|}{ Short-term stability } & \multicolumn{2}{|c|}{ Autosampler stability } & \multicolumn{2}{|c|}{$\begin{array}{l}\text { Freeze-thaw strability } \\
\text { (three cycles) }\end{array}$} & \multicolumn{2}{|c|}{ Long-term stability } \\
\hline & & & Accuracy (\%) & RSD (\%) & Accuracy (\%) & $\operatorname{RSD}(\%)$ & Accuracy (\%) & RSD (\%) & Accuracy (\%) & $\mathrm{RSD}(\%)$ \\
\hline \multirow[t]{27}{*}{ Plasma } & QCT & 1 & $100.23 \pm 1.69$ & 1.6 & $103.73 \pm 5.52$ & 5.32 & $98.27 \pm 6.33$ & 6.44 & $97.57 \pm 3.42$ & 3.51 \\
\hline & & 10 & $97.45 \pm 4.23$ & 4.34 & $99.34 \pm 6.73$ & 6.76 & $100.31 \pm 4.27$ & 4.26 & $96.41 \pm 5.79$ & 6.01 \\
\hline & & 100 & $95.97 \pm 2.36$ & 2.46 & $94.22 \pm 5.14$ & 5.46 & $98.35 \pm 7.84$ & 7.97 & $93.17 \pm 7.35$ & 7.89 \\
\hline & ISR & 1 & $99.24 \pm 6.68$ & 6.73 & $98.06 \pm 8.29$ & 8.45 & $103.15 \pm 5.43$ & 5.26 & $98.35 \pm 6.27$ & 6.38 \\
\hline & & 10 & $96.28 \pm 5.89$ & 6.12 & $97.72 \pm 3.83$ & 3.92 & $94.52 \pm 3.68$ & 3.89 & $96.44 \pm 6.33$ & 6.56 \\
\hline & & 100 & $102.18 \pm 4.95$ & 4.84 & $98.34 \pm 7.61$ & 7.74 & $99.63 \pm 3.38$ & 3.52 & $95.76 \pm 8.18$ & 8.54 \\
\hline & KMF & 1 & $96.50 \pm 6.47$ & 6.71 & $99.17 \pm 4.39$ & 4.43 & $101.23 \pm 8.76$ & 8.65 & $98.70 \pm 9.35$ & 9.47 \\
\hline & & 10 & $101.22 \pm 2.09$ & 2.06 & $102.65 \pm 5.28$ & 5.14 & $103.21 \pm 4.14$ & 4.01 & $100.48 \pm 7.44$ & 7.4 \\
\hline & & 100 & $97.10 \pm 3.69$ & 3.8 & $95.96 \pm 3.11$ & 3.24 & $94.24 \pm 6.63$ & 7.04 & $95.21 \pm 5.96$ & 6.26 \\
\hline & GA & 1 & $100.59 \pm 6.08$ & 6.04 & $97.28 \pm 2.19$ & 2.25 & $102.76 \pm 5.39$ & 5.25 & $98.92 \pm 5.81$ & 5.87 \\
\hline & & 10 & $102.54 \pm 7.33$ & 7.15 & $98.33 \pm 3.46$ & 3.52 & $98.42 \pm 2.84$ & 2.89 & $95.48 \pm 10.28$ & 10.77 \\
\hline & & 100 & $98.31 \pm 5.29$ & 5.39 & $96.83 \pm 8.30$ & 7.09 & $95.44 \pm 7.51$ & 7.87 & $92.19 \pm 8.81$ & 9.56 \\
\hline & GB & 1 & $96.95 \pm 2.87$ & 2.96 & $101.66 \pm 4.73$ & 8.57 & $96.53 \pm 5.48$ & 5.68 & $98.45 \pm 5.75$ & 5.84 \\
\hline & & 10 & $98.25 \pm 5.91$ & 6.01 & $95.45 \pm 5.97$ & 6.25 & $97.82 \pm 4.31$ & 4.41 & $95.72 \pm 6.59$ & 3.83 \\
\hline & & 100 & $98.74 \pm 4.06$ & 4.11 & $99.11 \pm 7.23$ & 7.3 & $96.55 \pm 5.94$ & 4.64 & $97.38 \pm 4.39$ & 6.88 \\
\hline & GC & 1 & $100.24 \pm 4.70$ & 3.1 & $98.49 \pm 6.22$ & 6.32 & $104.74 \pm 8.47$ & 8.09 & $100.07 \pm 10.14$ & 10.13 \\
\hline & & 10 & $104.11 \pm 3.38$ & 3.25 & $102.24 \pm 5.11$ & 5 & $100.15 \pm 4.52$ & 4.52 & $100.48 \pm 8.62$ & 8.58 \\
\hline & & 100 & $94.58 \pm 5.63$ & 5.96 & $91.17 \pm 5.33$ & 5.85 & $96.22 \pm 5.73$ & 5.96 & $93.97 \pm 4.38$ & 4.66 \\
\hline & BB & 1 & $98.10 \pm 7.83$ & 7.98 & $103.47 \pm 6.75$ & 6.52 & $99.85 \pm 6.27$ & 6.28 & $99.15 \pm 5.73$ & 5.78 \\
\hline & & 10 & $99.78 \pm 5.25$ & 5.26 & $95.29 \pm 2.77$ & 2.91 & $98.88 \pm 3.59$ & 3.63 & $96.38 \pm 8.63$ & 8.95 \\
\hline & & 100 & $94.05 \pm 2.61$ & 2.78 & $97.44 \pm 3.13$ & 3.21 & $95.47 \pm 4.92$ & 5.15 & $95.54 \pm 7.76$ & 8.12 \\
\hline & TSIIA & 1 & $96.50 \pm 6.47$ & 2.93 & $102.65 \pm 5.28$ & 4.33 & $100.31 \pm 4.27$ & 9.53 & $101.23 \pm 8.76$ & 3.47 \\
\hline & & 10 & $101.22 \pm 2.09$ & 3.41 & $95.96 \pm 3.11$ & 5.70 & $101.22 \pm 2.09$ & 7.26 & $103.21 \pm 4.14$ & 3.11 \\
\hline & & 100 & $95.96 \pm 3.11$ & 2.19 & $99.15 \pm 5.73$ & 4.92 & $95.97 \pm 2.36$ & 4.19 & $94.24 \pm 6.63$ & 3.58 \\
\hline & SAB & 5 & $97.28 \pm 2.19$ & 3.74 & $96.38 \pm 8.63$ & 6.51 & $99.24 \pm 6.68$ & 3.24 & $102.76 \pm 5.39$ & 4.25 \\
\hline & & 50 & $96.44 \pm 6.33$ & 4.33 & $96.41 \pm 5.79$ & 9.18 & $98.88 \pm 3.59$ & 7.45 & $95.72 \pm 6.59$ & 5.67 \\
\hline & & 500 & $95.76 \pm 8.18$ & 8.21 & $93.17 \pm 7.35$ & 6.88 & $95.47 \pm 4.92$ & 6.17 & $97.38 \pm 4.39$ & 7.69 \\
\hline
\end{tabular}

constituents in rat plasma. The correlation coefficients of the standard curves and the linear ranges of the plasma are listed in Table 4.

3.4.3 Precision and accuracy. The precision and accuracy data for six replicates of the QC samples at three concentrations are shown in Table 5 . The results demonstrated that the precision (RSD\%) of the method was $10.26 \%$.

3.4.4 Extraction recovery rates and matrix effects. The extraction recovery and matrix effects of each QC concentration are listed in Table 6. The extraction recoveries ranged from $76.86 \%$ to $95.77 \%$ at different concentrations in plasma. All ratios for matrix effects were in the range of 94.56-103.25\% in plasma samples. Thus, no significant matrix effects were observed for the analytes.

3.4.5 Stability. The data for freeze-thaw stability, shortterm temperature stability, long-term stability, and autosampler stability under different storage conditions are summarized in Table 7 . The results were well within the acceptable limits, therefore validating the established method for sample extraction, storage and intermittent analysis and indicating its suitability for pharmacokinetic study.

\subsection{Pharmacokinetics studies}

The developed and validated UPLC-QqQ-MS/MS method was successfully applied in the pharmacokinetics study of QCT, ISR,
$\mathrm{KMF}, \mathrm{GA}, \mathrm{GB}, \mathrm{GC}, \mathrm{BB}$, TSIIA, and SAB in rat plasma after an oral dose of $4.8 \mathrm{~g} \mathrm{~kg}^{-1} \mathrm{~d}^{-1}$ YDXNT. Fig. 7 shows the mean plasma concentration-time profiles of nine constituents in rat plasma. Table 8 shows the main pharmacokinetic parameters of QCT, ISR, KMF, GA, GB, GC, BB, TSIIA, and SAB in rat plasma.

GA and GB have similar pharmacokinetic parameters and may be connected to similar polar constituents, similar to the absorption and elimination processes. ${ }^{48}$ The $C_{\max }$ of GC was the lowest among the four ginkgolides, indicating that GC may be partially converted to hydrolyzed ginkgolide $\mathrm{C}$, as we detected. The time required to reach the maximum plasma concentration $\left(T_{\max }\right)$ was $0.75 \mathrm{~h}$ for GA, $1.00 \mathrm{~h}$ for GB, $1.50 \mathrm{~h}$ for GC, and $0.75 \mathrm{~h}$ for BB. The $T_{1 / 2}$ of four ginkgolides in YDXNT were $7.52 \mathrm{~h}$, $7.84 \mathrm{~h}, 5.45 \mathrm{~h}$, and $5.55 \mathrm{~h}$. Absorption of the four ginkgolides was slower than that of the flavonols, TSIIA and SAB, while their distribution occurred relatively faster than that of the flavonols, TSIIA and SAB. Additionally, the flavonols showed double peaks in the mean plasma concentration curves. This phenomenon has been reported previously, indicating that these components might have enterohepatic recirculation. ${ }^{\mathbf{4 9 , 5 0}}$ The double peaks suggested that enterohepatic recirculation, as well as intertransformation among the compounds, might have occurred. One possible explanation for this phenomenon is that some of the other compounds with similar structures might have transformed into these compounds. Since drug absorption is 

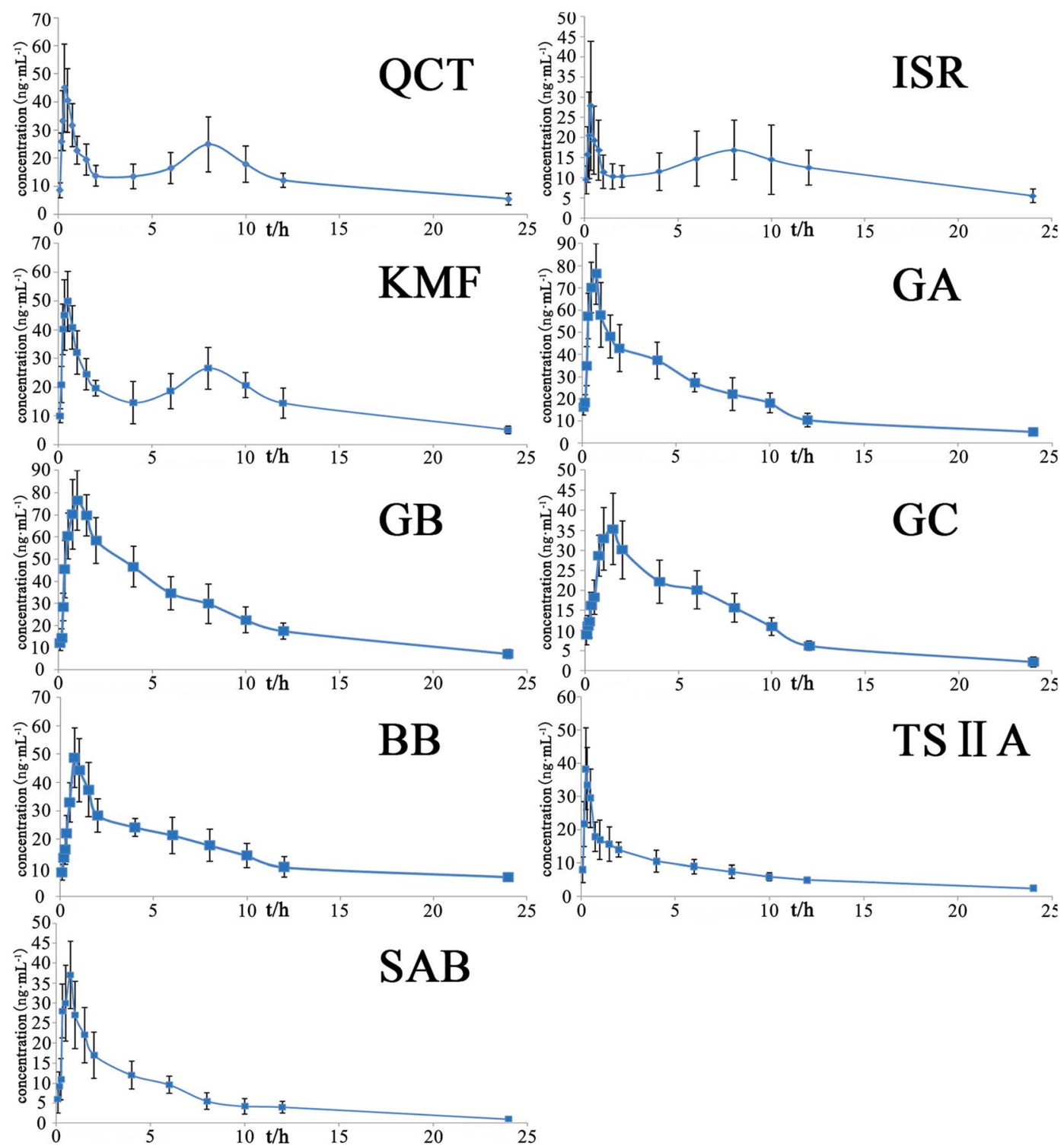

TS II A

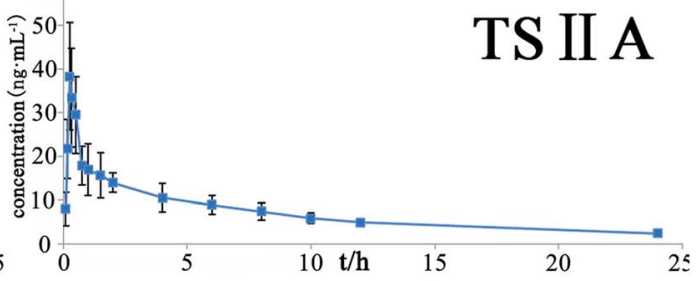

Fig. 7 Plasma concentration-time profiles of the analytes following oral administration of the YDXNT to rats (mean \pm SD, $n=6)$.

a complex process, more detailed adsorption studies are needed to ascertain the mechanism of the double-peak phenomenon.

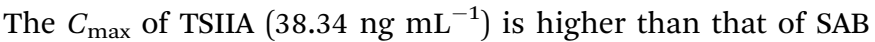
(32.00 ng $\left.\mathrm{mL}^{-1}\right)$, and the $T_{\max }$ and $T_{1 / 2}$ of TSIIA $(0.25 \mathrm{~h}, 7.04 \mathrm{~h})$ are lower than those of SAB $(0.75 \mathrm{~h}, 12.17 \mathrm{~h})$, indicating the absorption and distribution rates of TSIIA are faster than those of $\mathrm{SAB}$ in rats.

Table 8 PK parameters of the seven analytes following oral administration of YXC to rats $(n=6)$

\begin{tabular}{|c|c|c|c|c|c|c|}
\hline Analytes & $C_{\max } /\left(\mathrm{ng} \mathrm{mL}^{-1}\right)$ & $T_{\max }(\mathrm{h})$ & $\mathrm{AUC}_{0-t}\left(\mathrm{ng} \mathrm{mL}^{-1} \mathrm{~h}^{-1}\right)$ & $\operatorname{AUC}_{0-\mathrm{INF}}\left(\mathrm{ng} \mathrm{mL}^{-1} \mathrm{~h}^{-1}\right)$ & $T_{1 / 2}(\mathrm{~h})$ & $\operatorname{MRT}(\mathrm{h})$ \\
\hline QCT & $45.02 \pm 11.28$ & $0.33 \pm 0.11$ & $410.34 \pm 73.15$ & $412.46 \pm 82.67$ & $2.69 \pm 0.65$ & $8.44 \pm 1.24$ \\
\hline ISR & $27.85 \pm 8.38$ & $0.33 \pm 0.14$ & $319.17 \pm 49.27$ & $384.49 \pm 55.13$ & $8.19 \pm 2.42$ & $13.91 \pm 3.71$ \\
\hline GA & $76.31 \pm 18.19$ & $0.75 \pm 0.29$ & $490.92 \pm 72.63$ & $545.10 \pm 78.91$ & $7.52 \pm 2.08$ & $9.42 \pm 1.77$ \\
\hline GB & $76.54 \pm 15.43$ & $1.00 \pm 0.35$ & $610.18 \pm 91.45$ & $691.00 \pm 96.83$ & $7.84 \pm 3.53$ & $10.58 \pm 2.63$ \\
\hline GC & $35.35 \pm 10.28$ & $1.50 \pm 0.23$ & $281.80 \pm 41.33$ & $297.49 \pm 46.59$ & $5.45 \pm 1.48$ & $7.77 \pm 2.62$ \\
\hline $\mathrm{SAB}$ & $32.00 \pm 15.43$ & $0.75 \pm 0.18$ & $118.17 \pm 58.46$ & $135.72 \pm 64.24$ & $12.17 \pm 3.33$ & $9.46 \pm 2.37$ \\
\hline
\end{tabular}




\section{Conclusion}

In this research, we developed the UPLC-Q-TOF-MS method for analyzing and identifying the main absorbed constituents of YDXNT and their possible metabolites in rat plasma. A total of 52 constituents, including 44 prototype compounds and 8 metabolites, were identified or tentatively characterized. Among the prototype constituents, 15 flavonoids, 4 ginkgolides, 8 phenolic, 8 ginsenoside, and 7 diterpenoid tanshinones were identified. The metabolic routes of 8 metabolites in rat plasma after oral administration were hypothesized. The result indicated that the metabolites of YDXNT undergo the phase I metabolite pathway of hydrolysis (M2, M3, M4, and M6), dehydrogenation (M8) and the phase II metabolic route of glucuronide (M1, M5 and M7). Furthermore, a sensitive and reliable method based on UPLC-QqQ-MS was established for simultaneous quantification of nine abundant main compounds in rat plasma. The developed assay was successfully applied to assess the pharmacokinetics of ginkgetin aglycone, terpene lactones, salvianolic acid, and tanshinone from YDXNT in rat plasma. Our research clarifies for the first time the absorbed constituents, possible metabolites, and pharmacokinetics of YDXNT in vivo. This work provides more in-depth insight into the main active constituents of YDXNT in vivo as well as helpful information for clinical application. More importantly, our findings are helpful for better understanding the material foundation and action targets underlying the efficacy of YDXNT.

\section{Author contributions}

All the authors have approved the manuscript and agree with submission to your esteemed journal.

\section{Conflicts of interest}

There are no conflicts of interest to declare.

\section{Acknowledgements}

Financial support was provided by the Chinese drug discovery initiative project (No. 2012ZX09201201).

\section{References}

1 L. Y. Kong and R. L. Tan, Nat. Prod. Rep., 2015, 32, 16171621.

2 X. J. Wang, A. H. Zhang, H. Sun, Y. Han and G. L. Yan, TrAC, Trends Anal. Chem., 2016, 76, 86-94.

3 L. Ma, H. Liu, L. Meng, P. Qin, B. T. Zhang, Y. Y. Li, S. L. Man, Z. Liu, Z. X. Liu and A. P. Diao, RSC Adv., 2017, 7, 3408-3412. 4 X. J. Wang, A. H. Zhang, H. Sun and P. Wang, Am. J. Chin. Med., 2012, 40, 1109.

5 E. L. Leung, E. Clementi and W. S. Wong, Pharmacol. Res., 2017, 117, 65-66.

6 Y. Tao, H. Y. Xu, S. S. Wang, B. Wang, Y. C. Zhang, W. H. Wang, B. Huang, H. W. Wu, D. F. Li, Y. Zhang,
X. F. Xiao, Y. B. Li, H. J. Yang and L. Q. Huang, J. Chromatogr. B, 2013, 935, 1-9.

7 Z. Y. Tang, Y. Y. Hou, X. Y. Xu, A. N. Liu, L. F. Yau, T. T. Tong, Z. H. Jiang and G. Bai, RSC Adv., 2017, 7, 37459-37466.

8 M. Q. Jia, Y. J. Xiong, Y. Xue, Y. Wang and C. Yan, Molecules, 2017, 22, 1-17.

9 S. P. Wang, P. Fu, L. Liu, L. L. Wang, C. C. Peng, W. D. Zhang and R. H. Liu, RSC Adv., 2016, 6, 32811-32822.

$10 \mathrm{H}$. Wu and F. Fang, RSC Adv., 2016, 6, 28279-28288.

11 D. X. Chen, S. Lin, W. Xu, M. Q. Huang, J. F. Chu, F. Xiao, J. M. Lin and J. Peng, Molecules, 2015, 20, 18597-18619.

12 The State Pharmacopoeia Commission of PR China, The Pharmacopoeia of $P R$ China, China Medical Science and Technology Press, PR China, 2015.

13 J. L. Wang, L. Wang, L. Chen, X. J. Yin, H. Y. Xu, W. D. Wang and R. X. Liang, China J. Chin. Mater. Med., 2014, 9, 25472552.

14 L. Cheng, Y. Liu and X. Sun, J. Evidence-Based Complementary Altern. Med., 2017, 2017, 1-14.

$15 \mathrm{~J}$. Huang, The Second China-Japan-Korea Congress on the Blood Stasis Syndrome, 2003, pp. 61-64.

16 L. L. Gong, H. Y. Xu, L. Wang, X. J. Yin, H. J. Yuan, S. S. Wang, L. Cheng, X. J. Ma, S. R. Gao, R. X. Liang and H. J. Yang, J. Sep. Sci., 2016, 39, 611-622.

17 J. L. Wang, L. Wang, H. J. Yang, Y. You, H. Y. Xu, L. L. Gong, X. J. Yin, W. D. Wang, S. R. Gao, L. Cheng, R. X. Liang and F. L. Liao, BMC Complementary Altern. Med., 2015, 15, 109122.

18 W. D. Wang, L. Wang, H. J. Yang, J. L. Wang, X. J. Yin, H. Y. Xu, L. Cheng and R. X. Liang, J. Tradit. Chin. Med., 2014, 34, 699-709.

19 L. Cheng, G. F. Pan, X. D. Zhang, J. L. Wang, W. D. Wang, J. Y. Zhang, H. Wang, R. X. Liang and X. B. Sun, Sci. Rep., 2015, 5, 1-11.

20 J. B. Fourtillan, A. M. Brisson, J. Girault, I. Ingrand, J. P. Decourt, K. Drieu, P. Jouenne and A. Biber, Thérapie, 1995, 50, 137-144.

21 X. Li, C. Yu, Y. Lu, Y. Gu, J. Lu, W. Xu, L. Xuan and Y. Wang, Drug Metab. Dispos., 2007, 35, 234-239.

22 Z. Ouyang, M. Zhao, J. Tang and L. Pan, Pharmacogn. Mag., 2012, 8, 256.

23 S. Li, X. Jiang, Q. Yang and Z. Jin, J. Biomed. Eng., 2003, 20, 692-694.

24 L. Q. Sun, Asian Journal of Drug Metabolism \& Pharmacokinetics, 2002, 2, 291-298.

25 Q. Chen, Z. Liang, E. Brand, H. Chen and Z. Zhao, Planta Med., 2017, 82, 263-272.

26 W. J. Chen, Y. Y. Liu, M. Y. Wei, L. Q. Shi, Y. Wu, Z. Y. Liu, S. Liu, F. R. Song and Z. Q. Liu, RSC Adv., 2017, 7, 4296442972.

27 G. Y. Ding, Y. S. Wang, A. N. Liu, Y. Y. Hong, T. J. Zhang, G. Bai and C. X. Liu, RSC Adv., 2017, 7, 22034-22044.

28 J. W. Lee, H. J. Mok, D. Y. Lee, S. C. Park, G. S. Kim, S. E. Lee, Y. S. Lee, K. P. Kin and H. D. Kim, J. Proteome Res., 2017, 16, 1460-1469.

29 J. H. Lee, H. Shinohara, J. H. Jung, H. J. Mok, Y. Akao and K. P. Kim, RSC Adv., 2016, 6, 29512-29518. 
30 US Department of Health and Human Services, Guidance for Industry Analytical Procedures and Methods Validation for Drugs and Biologics, 2015, http:/www.fda.gov/downloads/ drugs/guidancecomplianceregulatoryinformation/ guidances/ucm386366.pdf.

31 H. Wang, X. Bai, J. H. Sun, Y. Kano, T. Makino and D. Yuan, Planta Med., 2013, 79, 1552-1557.

32 Y. F. Zhang, P. Y. Shi, H. Yao, Q. Shao and X. H. Fan, Curr. Drug Metab., 2012, 13, 510-523.

33 M. Xu, Z. C. Zhang, G. Fu, S. F. Sun, J. H. Sun, M. Yang, A. H. Liu, J. Han and D. A. Guo, J. Chromatogr. B, 2007, 856, 100-107.

34 X. Zhang, D. H. Yang, F. Xu, S. Huang, L. Zhang, G. X. Liu and S. Q. Cai, Biomed. Chromatogr., 2015, 29, 285-304.

35 D. L. Wang, Y. Liang, W. D. Chen, L. Xue, G. J. Wang and X. D. Liu, Acta Pharmacol. Sin., 2008, 29, 376-384.

36 X. G. Liu, L. D. Qi, Z. Y. Fan, X. Dong, R. Z. Guo, F. C. Lou, S. Fanali, P. Li and H. Yang, J. Chromatogr. A, 2015, 1388, 251-258.

37 S. Ding, E. Dudley, S. Plummer, J. Tang, R. P. Newton and A. G. Brenton, Phytochemistry, 2008, 69, 1555-1564.

38 X. J. Liu, K. Yang, G. Du, L. Xu and K. Lan, Anal. Bioanal. Chem., 2015, 407, 7945-7956.

39 Y. Liu, M. L. Bandu and H. Desaire, Anal. Chem., 2005, 77, 6655-6663.
40 H. Cai, D. J. Boocock, W. P. Steward and A. J. Gescher, Cancer Chemother. Pharmacol., 2007, 60, 257-266.

41 H. L. Ma, Y. Liu, X. Mai, Y. J. Liao, K. Zhang, B. Liu, X. Xie and Q. L. Du, J. Pharm. Biomed. Anal., 2016, 125, 194-204.

42 Y. J. Shi, Y. Wang, H. Z. Zhu, H. X. Zheng, W. D. Zhu, J. Xu and M. Y. Li, Chin. J. Pharm. Anal., 2015, 35, 1417-1423.

43 Y. Z. Zhang, T. Wang, S. L. Zhou, H. L. Liu and C. Yan, China Pharmacy, 2016, 27, 479-482.

44 J. H. Ding, X. X. Wang, H. Zhang, S. S. Pan, M. B. Luo, J. Q. Li and H. W. Chen, Chem. J. Chin. Univ., 2011, 32, 1714-1719.

45 J. H. Sun, M. Yang, X. M. Wang, M. Xu, A. H. Liu and D. A. Guo, J. Pharmaceut. Biomed., 2007, 44, 564-574.

46 P. Li, G. J. Wang, J. Li, H. P. Hao and C. N. Zheng, J. Mass Spectrom., 2006, 41, 670-684.

47 P. Li, G. J. Wang, J. Li, H. P. Hao and C. N. Zheng, Rapid Commun. Mass Spectrom., 2006, 20, 815-822.

48 Z. Yan-Yan, G. Li-Li, S. Guo-Ming, R. Rong and T. Jing-Zhen, Drug Res., 2016, 66, 520-526.

49 Z. P. Chen, J. Sun, H. X. Chen, Y. Y. Xiao, D. Liu, J. Chen, H. Cai and B. C. Cai, Fitoterapia, 2010, 81, 1045-1052.

50 W. P. Wang, N. Liu, Q. Kang, P. P. Du, Y. Lan, B. C. Zhao, Y. Y. Chen, Q. Zhang, H. Li, Y. W. Zhang and Q. Wu, J. Zhejiang Univ., Sci., B, 2014, 15, 929-939. 